\title{
Virtual Visitation: Exploring the Impact on Patients and Families During COVID-19 and Beyond
}

\author{
Sven Gierlinger, CXO, Julia Olsheski, PhD, Steven Caiola, MS, LSSB, \\ and Nicole Giammarinaro, MSN, RN, CPXP
}

Objective: Northwell Health, New York's largest health care organization, rapidly adopted technology solutions to support patient and family communication during the COVID-19 pandemic.

Methods: This case series outlines the pragmatic, interdisciplinary approach Northwell underwent to rapidly implement patient virtual visitation processes during the peak of the initial crisis.

Results: Implementation of large-scale virtual visitation required leadership, technology, and dedicated, empathetic frontline professionals. Patient and family feedback uncovered varied feelings and perspectives, from confusion to gratitude.

Conclusion: Subsequent efforts to obtain direct patient and family perspectives and insights helped Northwell identify areas of strength and ongoing performance improvement.

Keywords: virtual visitation; COVID-19; technology; communication; patient experience.

\footnotetext{
T
} he power of human connection has become increasingly apparent throughout the COVID-19 pandemic and subsequent recovery phases. Due to the need for social distancing, people worldwide have turned to virtual means of communication, staying in touch with family, friends, and colleagues via digital technology platforms. On March 18, 2020, the New York State Department of Health (NYSDOH) issued a health advisory, suspending all hospital visitation. ${ }^{1}$ As a result, hospitals rapidly transformed existing in-person visitation practices to meet large-scale virtual programming needs.

Family members often take on various roles-such as advocate, emotional support person, and postdischarge caregiver-for an ill or injured loved one. ${ }^{2}$ The Institute for Patient- and Family-Centered Care, a nonprofit organization founded in 1992, has been leading a cultural transformation where families are valued as care partners, as opposed to "visitors."3 Although widely adopted and well-received in specialized units, such as neonatal intensive care units, ${ }^{4}$ virtual visitation had not been widely implemented across adult care settings. The NYSDOH guidance therefore required organizational leadership, innovation, flexibility, and systems ingenuity to meet the evolving needs of patients, families, and health care professionals. An overarching goal was ensuring patients and families were afforded opportunities to stay connected throughout hospitalization.

Reflecting the impact of COVID-19 surges, hospital environments became increasingly depersonalized, with health care providers wearing extensive personal protective equipment (PPE) and taking remarkable measures to socially distance and minimize exposure. Patients' room doors were kept primarily closed, while codes and alerts blared in the halls overhead. The lack of families and visitors became increasingly obvious, aiding feelings of isolation and confinement. With fear of nosocomial transmission, impactful modalities (such as sitting at the bedside) and empathetic, therapeutic touch were no longer taking place.

With those scenarios-common to so many health care systems during the pandemic-as a backdrop, comes our experience. Northwell Health is the largest health care system in New York State, geographically spread throughout New York City's 5 boroughs, Westchester County, and Long Island. With 23 hospitals,

From Northwell Health, Lake Success, NY. 
approximately 820 medical practices, and over 72000 employees, Northwell has cared for more than 100000 COVID-positive patients to date. This case series outlines a pragmatic approach to implementing virtual visitation during the initial peak and obtaining patient and family perspectives to help inform performance improvement and future programming.

\section{Methods}

\section{Implementing virtual visitation}

Through swift and focused multidisciplinary collaboration, numerous Northwell teams came together to implement large-scale virtual visitation across the organization during the first wave of the COVID crisis. The initial priority involved securing devices that could support patientfamily communication. Prior to COVID, each facility had only a handful of tablets that were used primarily during leadership rounding, so once visitation was restricted, we needed a large quantity of devices within a matter of days. Through diligent work from System Procurement and internal Foundation, Northwell was able to acquire nearly 900 devices, including iPads, PadlnMotion tablets, and Samsung tablets.

Typically, the benefits of using wireless tablets within a health care setting include long battery life, powerful data processing, advanced operating systems, large screens, and easy end-user navigation. ${ }^{4}$ During COVID-19 and its associated isolation precautions, tablets offered a lifeline for effective and socially distant communication. With new devices in hand, the system Office of the Chief Information Officer (OClO) and site-based Information Technology (IT) teams were engaged. They worked tirelessly to streamline connectivity, download necessary apps, test devices on approved WiFi networks, and troubleshoot issues. Once set up, devices were strategically deployed across all Northwell hospitals and post-acute rehabilitation facilities.

Frontline teams quickly realized that a model similar to mobile proning teams, who focus solely on turning and positioning COVID patients to promote optimal respiratory ventilation, ${ }^{5}$ was needed to support virtual visitation. During the initial COVID wave, elective surgeries were not permissible, as per the NYSDOH. As a result, large numbers of clinical and nonclinical ambulatory surgery employees were redeployed throughout the organization, with many assigned and dedicated to facilitating newly created virtual visitation processes. These employees were primarily responsible for creating unit-based schedules, coordinating logistics, navigating devices on behalf of patients, being present during video calls, and sanitizing the devices between uses. Finally, if necessary, virtual interpretation services were used to overcome language barriers between staff and patients.

What began as an ad hoc function quickly became a valued and meaningful role. Utilizing triage mentality, virtual visitation was first offered during unit-based rounding protocols to those patients with the highest acuity and need to connect with family. We had no formal script; instead, unit-based leaders and frontline team members had open dialogues with patients and families to gauge their interest in virtual visitation. That included patients with an active end-of-life care plan, critically ill patients within intensive care units, and those soon to be intubated or recently extubated. Utilization also occurred within specialty areas such as labor and delivery, pediatrics, inpatient psychiatry, medical units, and long-term rehab facilities. Frontline teams appreciated the supplementary support so they could prioritize ongoing physical assessments and medical interventions. Donned in PPE, virtual visitation team members often served as physical extensions of the patient's loved ones-holding their hand, offering prayers, and, at times, bearing witness to a last breath. In reflecting on that time, this role required absolute professionalism, empathy, and compassion.

In summer 2020, although demand for virtual visitation was still at an all-time high when ambulatory surgery was reinstated, redeployed staff returned to their responsibilities. To fill this void without interruption to patients and their families, site leaders quickly pivoted and refined processes and protocols utilizing Patient \& Customer Experience and Hospitality department team members. Throughout spring 2021, the NYSDOH offered guidance to open in-person visitation, and the institution's Clinical Advisory Group has been taking a pragmatic approach to doing that in a measured and safe manner across care settings.

\section{Listening to the 'voice' of patients and families}

Our institution's mission is grounded in providing "quality service and patient-centered care." Honoring those 
tenets, during the initial COVID wave, the system "Voice of the Customer End User Device Workgroup" was created with system and site-based interdisciplinary representation. Despite challenging and unprecedented times, conscious attention and effort was undertaken to assess the use and impact of virtual devices. One of the major work streams was to capture and examine patient and family thoughts, feedback, and the overall experience as it relates to virtual visitation.

The system Office of Patient \& Customer Experience (OPCE), led by Sven Gierlinger, SVP Chief Experience Officer, reached out to our colleagues at Press Ganey to add a custom question to patient experience surveys. Beginning on December 1, 2020, discharged inpatients were asked to rate the "Degree to which you were able to stay connected with your family/caregiver during your stay." Potential answers include the Likert scale responses of Always, Usually, Sometimes, and Never, with "Always" representing the Top Box score. The OPCE team believes these quantitative insights are important to track and trend, particularly since in-person and virtual visitation remain in constant flux.

In an effort to obtain additional, focused, qualitative feedback, OPCE partnered with our institution's Digital Patient Experience (dPX) colleagues. The approach consisted of voluntary, semistructured, interview-type conversations with patients and family members who engaged in virtual visitation multiple times while the patient was hospitalized. OPCE contacted site-based Patient Experience leads, also known as Culture Leaders, at 3 hospitals, asking them to identify potential participants. This convenience sample excluded instances where the patient passed away during and/or immediately following hospitalization.

The OPCE team phoned potential interview candidates to make a personalized connection, explain the purpose of the interviews, and schedule them, if interested. For consistency, the same Digital Customer Experience Researcher on the dPX team facilitated all sessions, which were 30-minute, semiscripted interviews conducted virtually via Microsoft Teams. The tone was intentionally conversational so that patients and family members would feel comfortable delving into themes that were most impactful during their experience. After some initial ice breakers, such as "What were some of your feelings about being a patient/having a loved one in the hospital during the early days of the COVID-19 pandemic?" we moved on to some more pragmatic, implementation questions and rating scales. These included questions such as "How did you first learn about the option for virtual visitation? Was it something you inquired about or did someone offer it to you? How was it explained to you?" Patients were also asked, on a scale of 1 (easy) to 5 (difficult), to rate their experience with the technology aspect when connecting with their loved ones. They also provided verbal consent to be recorded and were given a $\$ 15$ gift card upon completion of the interview.

Transcriptions were generated by uploading the interview recordings to a platform called UserTesting. In addition to these transcriptions, this platform also allowed for a keyword mapping tool that organized high-level themes and adjectives into groupings along a sentiment axis from negative to neutral to positive. Transcripts were then read carefully and annotated by the Digital Customer Experience Researcher, which allowed for strengthening of some of the automated themes as well as the emergence of new, more nuanced themes. These themes were organized into those that we could address with design and/or procedure updates (actionable insights), those that came up most frequently overall (frequency), and those that came up across our 3 interview sessions (commonality).

This feedback, along with the responses to the new Press Ganey question, was presented to the system Voice of the Customer End User Device Workgroup. The results led to robust discussion and brainstorming regarding how to improve the process to be more patient-centered. Findings were also shared with our hospital-based Culture Leaders. As many of their local strategic plans focused on patient-family communication, this information was helpful to them in considering plans for expansion and/or sustaining virtual visitation efforts. The process map in the Figure outlines key milestones within this feedback loop.

\section{Outcomes}

During the height of the initial COVID-19 crisis, virtual visitation was a new and ever-evolving process. Amidst the chaos, mechanisms to capture the quantity and quality of 


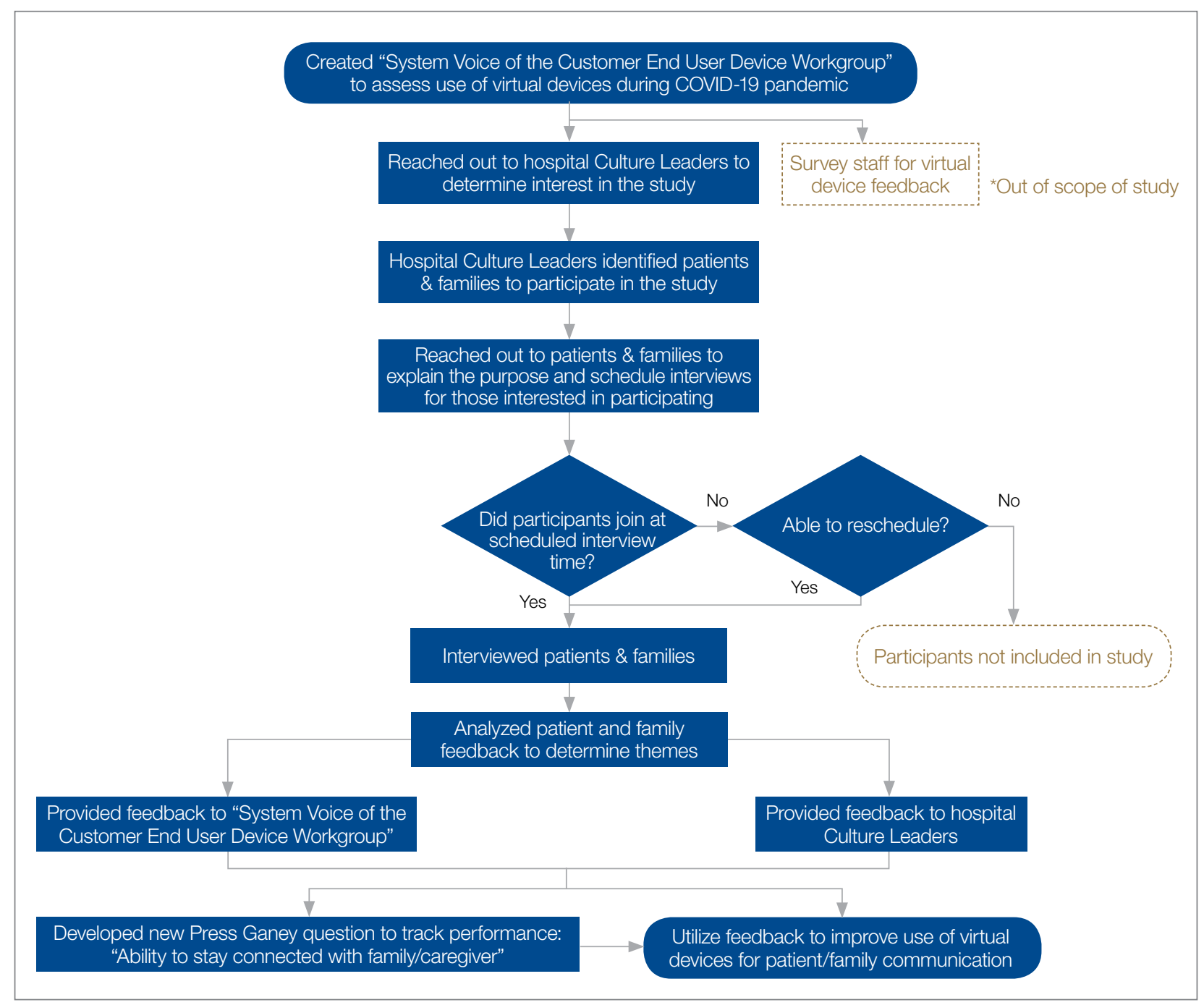

Figure. Process map to obtain patient feedback and share it with key stakeholders for process improvement.

virtual visits were not in place. Based on informal observation, a majority of patients utilized personal devices to connect with loved ones, and staff even offered their own cellular devices to facilitate timely patient-family communication. The technology primarily used included FaceTime, Zoom, and EZCall, as there was much public awareness and comfort with those platforms.

In the first quarter of 2021, our institution overall performed at a Top Box score of 60.2 for our ability to assist patients with staying connected to their family/caregiver during their inpatient visit. With more than 6700 returned surveys during that time period, our hospitals earned Top Box scores ranging between 48.0 and 75.3. At this time, obtaining a national benchmark ranking is not possible, because the question regarding connectedness is unique to Northwell inpatient settings. As other health care organizations adopt this customized question, further peer-to-peer measurements can be established.

Regarding virtual interviews, 25 patients were initially contacted to determine their interest in participating. Of that sample, 17 patients were engaged over the phone, representing a reach rate of $68 \%$. Overall, 10 interviews were scheduled; 7 patients did not show up, resulting in 3 completed interviews. During follow-up, "no-show" participants either gave no response or stated they had a conflict at their originally scheduled time but were not interested 
in rescheduling due to personal circumstances. Through such conversations, ongoing health complications were found to be a reoccurring barrier to participation.

Each of the participating patients had experienced being placed on a ventilator. They described their hospitalization as a time of "confusion and despair" in the first days after extubation. After we reviewed interview recordings, a reoccurring theme across all interviews was the feeling of gratitude. Patients expressed deep and heartfelt appreciation for being given the opportunity to connect as a family. One patient described virtual visitation sessions as her "only tether to reality when nothing else made sense."

Interestingly enough, none of the participants knew that virtual visitation was an option and/or thought to inquire about it before a hospital staff member offered to set up a session. Patients recounted how they were weak and physically unable to connect to the sessions without significant assistance. They reported examples of not having the physical strength to hold up the tablet or needing a staff member to facilitate the conversation because the patient could not speak loudly enough and/or they were having difficulty hearing over background medical equipment noises. Participants also described times when a nurse or social worker would stand and hold the tablet for 20 to 30 minutes at a time, further describing mixed feelings of gratitude, guilt for "taking up their time," and a desire for more privacy to have those precious conversations.

\section{Discussion}

Our institution encountered various barriers when establishing, implementing, and sustaining virtual visitation. The acquisition and bulk purchasing of devices, so that each hospital unit and department had adequate par levels during a high-demand time frame, was an initial challenge. Ensuring appropriate safeguards, software programming, and access to WiFi required ingenuity from IT teams. Leaders sought to advocate for the importance of prioritizing virtual visitation alongside clinical interventions. For team members, education was needed to build awareness, learn how to navigate technology, and troubleshoot, in real-time, issues such as poor connectivity. However, despite these organizational struggles, the hospital's frontline professionals fully recognized and understood the humanistic value of connecting ill patients with their loved ones. Harnessing their teamwork, empathy, and innovative spirits, they forged through such difficulties to create meaningful interactions.

Although virtual visitation occurred prior to the COVID-19 pandemic, particularly in subspecialty areas such as neonatal intensive care units, ${ }^{6}$ it was not commonplace in most adult inpatient care settings. However, now that virtual means to communication are widely accepted and preferred, our hospital anticipates these offerings will become a broad patient expectation and, therefore, part of standard hospital care and operations. Health care leaders and interdisciplinary teams must therefore prioritize virtual visitation protocols, efforts, and future programming. It is no longer an exception to the rule, but rather a critical approach when ensuring quality communication between patients, families, and care teams.

We strive to continually improve by including user feedback as part of an interactive design process. For a broader, more permanent installation of virtual visitation, health care organizations must proactively promote this capability as a valued option. Considering health literacy and comfort with technology, functionality, and logistics must be carefully explained to patients and their families. This may require additional staff training so that they are knowledgeable, comfortable with, and able to troubleshoot questions/concerns in real time. There needs to be an adequate number of mobile devices available at a unit or departmental level to meet short-term and long-term demands. Additionally, now that we have emerged from our initial crisis-based mentality, it is time to consider alternatives to alleviate the need for staff assistance, such as mounts to hold devices and enabling voice controls.

\section{Conclusion}

As an organization grounded in the spirit of innovation, Northwell has been able to quickly pivot, adopting virtual visitation to address emerging and complex communication needs. Taking a best practice established during a crisis period and engraining it into sustainable organizational culture and operations requires visionary leadership, strong teamwork, and an unbridled commitment 
to patient and family centeredness. Despite unprecedented challenges, our commitment to listening to the "voice" of patients and families never wavered. Using their insights and feedback as critical components to the decision-making process, there is much work ahead within the realm of virtual visitation.

Acknowledgements: The authors would like to acknowledge the Northwell Health providers, frontline health care professionals, and team members who worked tirelessly to care for its community during initial COVID-19 waves and every day thereafter. Heartfelt gratitude to Northwell's senior leaders for the visionary leadership; the OCIO and hospital-based IT teams for their swift collaboration; and dedicated Culture Leaders, Patient Experience team members, and redeployed staff for their unbridled passion for caring for patients and families. Special thanks to Agnes Barden, DNP, RN, CPXP, Joseph Narvaez, MBA, and Natalie Bashkin, MBA, from the system Office of Patient \& Customer Experience, and Carolyne Burgess, MPH, from the Digital Patient Experience teams, for their participation, leadership, and syngeristic partnerships.

Corresponding Author: Nicole Giammarinaro, MSN, RN, CPXP, Director, Patient \& Customer Experience, Northwell Health, 2000
Marcus Ave, Lake Success, NY 11042; nfilippa@northwell.edu.

Financial disclosures: Sven Gierlinger serves on the Speakers Bureau for Northwell Health and as an Executive Board Member for The Beryl Institute.

doi:10.12788/jcom.0064

\section{References}

1. New York State Department of Health. Health advisory: COVID19 guidance for hospital operators regarding visitation. March 18, 2020. https://coronavirus.health.ny.gov/system/files/documents/2020/03/covid19-hospital-visitation-guidance-3.18.20.pdf

2. Zhang $Y$. Family functioning in the context of an adult family member with illness: a concept analysis. J Clin Nurs. 2018;27(15-16):32053224. doi:10.1111/jocn. 14500

3. Institute for Patient- \& Family-Centered Care. Better Together: Partnering with Families. https://www.ipfcc.org/bestpractices/better-together-ny.html

4. Marceglia S, Bonacina S, Zaccaria V, et al. How might the iPad change healthcare? J R Soc Med. 2012;105(6):233-241. doi:10.1258/jrsm.2012.110296

5. Short B, Parekh M, Ryan P, et al. Rapid implementation of a mobile prone team during the COVID-19 pandemic. J Crit Care. 2020;60:230-234. doi:10.1016/j.jcrc.2020.08.020

6. Yeo C, Ho SK, Khong K, Lau Y. Virtual visitation in the neonatal intensive care: experience with the use of internet and telemedicine in a tertiary neonatal unit. Perm J. 2011;15(3):32-36. 